BARD Final report Eshed and Hake 
BARD Final report Eshed and Hake

Final Scientific Report - Cover Page (G6a)

BARD Project Number: IS-4536-12C

Title: Shaping plant architecture by age dependent programs: implications for food, feed and biofuel

Start Date of Project: 12-2012

Date of Submission of the report: $\mathbf{1 0 - 2 0 1 5}$

\begin{tabular}{|l|l|}
\hline Investigators & Affiliated Institutions \\
\hline Yuval Eshed & Weizmann Institute of Science \\
\hline Sarah Hake & PGEC, USDA, Albany, CA \\
\hline & \\
\hline & \\
\hline
\end{tabular}

Keywords not appearing in the title and in order of importance. Avoid abbreviations.

Side shoots

Lignification

Storage organs

Abbreviations commonly used in the report, in alphabetical order:

miR - micro RNA

LOG - Lonely Guy

Budget: IS: \$243K US: \$135K

Total: \$378K

Signature

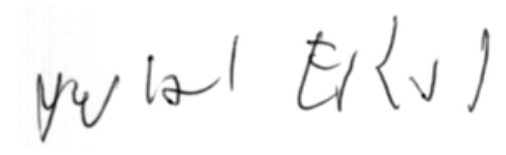

Principal Investigator (PI) Yuval Eshed

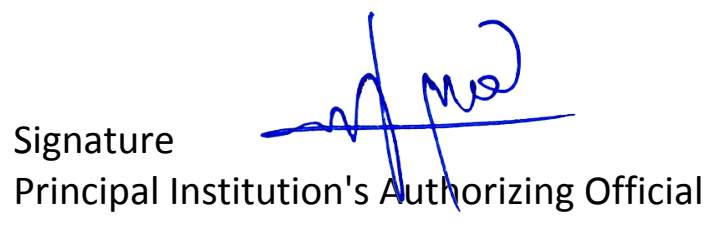

Dr. Doron Amit, Head Research Grants and Projects Office Weizmann Institute of Science

Rehovot 76100 , Israel 
BARD Final report Eshed and Hake

Final Scientific Report - Publication Summary (numbers) (G6b)

\begin{tabular}{|c|c|c|c|c|}
\hline & $\begin{array}{c}\text { Joint } \\
\text { IS/US } \\
\text { authorship }\end{array}$ & $\begin{array}{l}\text { US Authors } \\
\text { only }\end{array}$ & $\begin{array}{l}\text { Israeli } \\
\text { Authors } \\
\text { only }\end{array}$ & Total \\
\hline $\begin{array}{l}\text { Refereed (published, in press, accepted) } \\
\text { BARD support acknowledged }\end{array}$ & & 1 & 2 & 3 \\
\hline Submitted, in review, in preparation & & 1 & 1 & 2 \\
\hline \multicolumn{5}{|l|}{ Invited review papers } \\
\hline \multicolumn{5}{|l|}{ Book chapters } \\
\hline \multicolumn{5}{|l|}{ Books } \\
\hline \multicolumn{5}{|l|}{ Master theses } \\
\hline \multicolumn{5}{|l|}{ Ph.D. theses } \\
\hline \multicolumn{5}{|l|}{ Abstracts } \\
\hline Not refereed (proceedings, reports, etc.) & & & & \\
\hline
\end{tabular}

Postdoctoral Training: List the names and social security/identity numbers of all postdocs who received more than $50 \%$ of their funding by the grant.

Cooperation Summary (numbers)

\begin{tabular}{|l|l|l|l|l|}
\hline & $\begin{array}{c}\text { From US to } \\
\text { Israel }\end{array}$ & $\begin{array}{c}\text { From Israel } \\
\text { to US }\end{array}$ & $\begin{array}{c}\text { Together, } \\
\text { elsewhere }\end{array}$ & Total \\
\hline $\begin{array}{l}\text { Short Visits \& } \\
\text { Meetings }\end{array}$ & & 2 & & 2 \\
\hline $\begin{array}{l}\text { Longer Visits } \\
\text { (Sabbaticals) }\end{array}$ & 1 & & & $1(2016)$ \\
\hline
\end{tabular}

Description of Cooperation:

See at the bottom of the report

Patent Summary (numbers)

\begin{tabular}{|l|l|l|l|l|}
\hline & $\begin{array}{c}\text { Israeli } \\
\text { inventor } \\
\text { only }\end{array}$ & $\begin{array}{c}\text { US inventor } \\
\text { only }\end{array}$ & $\begin{array}{c}\text { Joint } \\
\text { IS/US } \\
\text { inventors }\end{array}$ & Total \\
\hline Submitted & & 1 & & 1 \\
\hline $\begin{array}{l}\text { Issued } \\
\text { (allowed) }\end{array}$ & & & & \\
\hline Licensed & & & & \\
\hline
\end{tabular}


Shaping plant architecture by age dependent programs: implications for food, feed and biofuel - Final Scientific report.

Age dependent programs are responsible for the physiological and developmental differences of young and mature plants. These include a range of morphological characters such as leaf shape and leaf composition (waxes, lignin etc..) but also different in developmental potentials. Apical buds of juvenile plants are vegetative, while those of mature plants can be reproductive. Likewise, basal buds form in the axills of juvenile leaves have different fates than distal buds formed in the axils of mature leaves. The goal of our joint project is to understand and exploit theses age related programs for specific improvement of crop plants.

To that end both the WIS group and the PGEC group are using mutants with age related defects as well as modified expression of miR156 to modify age related programs in crop plants- Tomato and potato in Israel and Maize, switchgrass and Brchipodium in the US. In the US, major effort were made to:

A) Characterize the contribution of selected miR156 target genes to yield component traits of maize.

B) Functional analysis of microRNAs and their targets in new crop plants.

In Israel, the research progressed in several directions:

C) Understanding the interplay between age dependent programs and the potential of tomato and potato meristems to produce tubers.

D) Evaluation of the agronomic value of mutants that alter flowering regime in side shoots in general, and in the sympodial buds in particular

E) Characterization of wild type axillary buds, comparing shoot ontogeny of gradually maturing apices from basal and distal positions along the main shoot of tomato.

\section{Resulting publications:}

Eviatar-Ribak T, Shalit-Kaneh A, Chappell-Maor L, Amsellem Z, Eshed Y, Lifschitz E. (2013). A cytokinin-activating enzyme promotes tuber formation in tomato. Curr Biol. 23(12): 1057-64.

Park SJ, Jiang K, Tal L, Yichie Y, Gar O, Zamir D, Eshed Y, Lippman ZB. (2014). Optimization of crop productivity in tomato using induced mutations in the florigen pathway. Nat Genet. 46(12): 1337-42.

Chuck, G., Brown, P., Meeley, R., Hake, S. (2014). Maize SBP-box transcription factors unbranched2 and unbranched3 affect yield traits by regulating the rate of lateral primordia initiation. PNAS, 111:18775-80.

\section{A) Understanding the functions of MIR156 targets in maize}

One of our main objectives was to understand the biological roles of the MIR156 targets in maize. Using reverse genetics we identified several loss of function mutations in three targets of MIR156. The first, tasselsheath4 (tsh4) (Chuck et al 2010) (Fig 1A) was shown to be 
BARD Final report Eshed and Hake

important for suppressing growth of lateral organs such as bracts during the floral phase of maize. We also demonstrated that a pair duplicated MIR156 target genes related to tsh4 called unbranched2 (ub2) and unbranched ( $u b 3$ ) function as redundant factors that limit the rate of cell differentiation to the lateral domains of meristems (Chuck et al 2014). In particular, ub3 may have played a major role in improving maize grain yields since it co-localizes to QTL important for ear row number, and unbranched double mutants make many extra kernel rows (Fig 1B). In support of this concept, dominant mutations in the microRNA binding sites of the rice orthologue of $u b 3$ lead to the WEALTHY FARMERS PANICLE phenotype that increases grain yields. Finally, triple mutants of tsh4/ub2/ub3 make many extra tillers and leaves demonstrating that these genes may be important for repression of lateral organ growth (Fig 1C), and thus may be useful for increasing biomass levels. Based on these results, we believe that these genes form a specific clade of MIR156 targets that may be useful for improving the agronomic qualities of crop plants as described later.
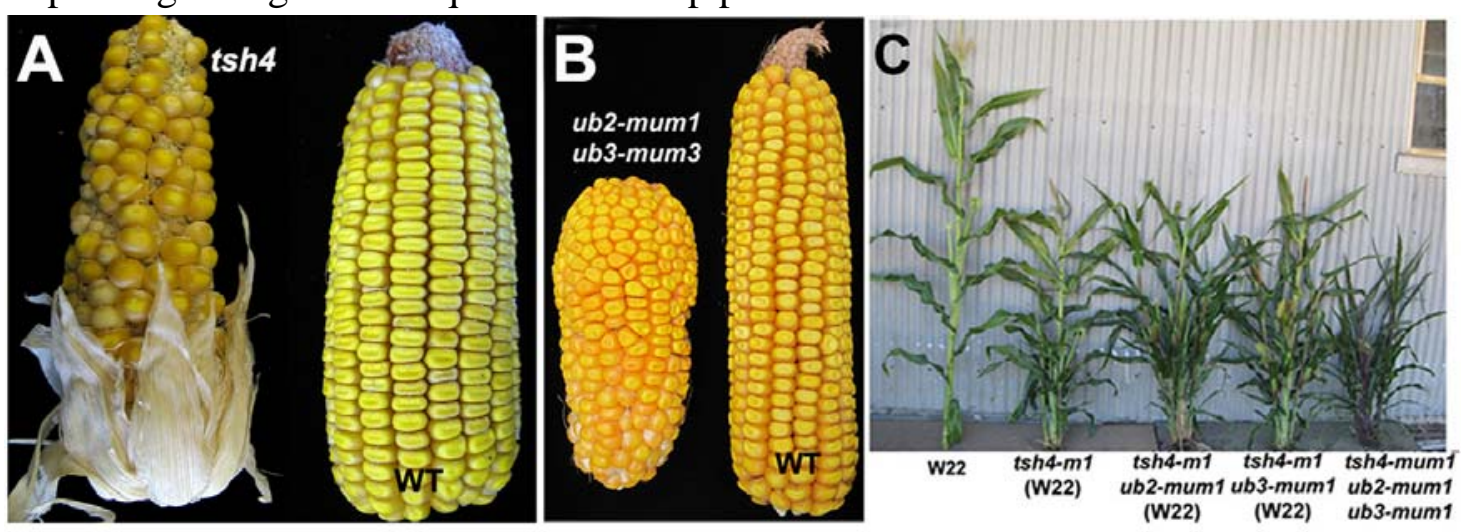

Figure 1. phenotypes of MIR156 target mutants

1A) tasselsheath4 ear (left) compared to wild type. 1B) unbranched2/3 double mutant ear (left) compared to wild type. 1C) Tillering phenotypes of tasselsheath4, unbranched 2/3 double and triple mutants showing increased tillering.

The following publication describing this project was supported by the last round of BARD funding:

Chuck, G., Brown, P., Meeley, R., and Hake, S. (2014). The maize SBP-box transcription factors unbranched 2 and unbranched 3 affect yield traits by regulating the rate of lateral primordia initiation. Proc Natl Acad Sci; 111:18775-80.

The previous round of BARD funding supported the following publication:

Chuck, G., Whipple, C., Jackson, D. and Hake, S. (2010). The maize SBP-box transcription factor tasselsheath4 regulates bract development and establishment of meristem boundaries. Development. 137(8): 1243-50.

\section{B) Functional analysis of microRNAs and their targets in new crop plants}


BARD Final report Eshed and Hake

In order to test our hypothesis that MIR156 microRNAs or their targets can be useful agronomically for developing new biofuels or new food sources, new transformation protocols were necessary. We developed a transformation protocol based on meristem tissue culture and showed that it can be used to transform a potential biofuel crop called Big Bluestem (Fig 2A), a current food crop called foxtail millet (Fig 2B), and finally, an orphan African food crop called teff (Fig 2C).

A manuscript describing our transformation protocol is under preparation.

The following US patent application derived from this work was filed on 09/25/2013:

Chuck et al., Overexpression of Cg1 Improves Biofuel Properties of Switchgrass

Docket No. 0155.09, Serial No. 14/036,844

The previous round of BARD funding supported the following publication:

Chuck G., Tobias C, Sun L, Kraemer F, Li C, Dibble D, Arora R, Bragg JN, Vogel JP, Singh S, Simmons BA, Pauly M, Hake S. (2011). Overexpression of the maize Corngrass1 microRNA prevents flowering, improves digestibility, and increases starch content of switchgrass. Proc Natl Acad Sci. 108:17550-5.
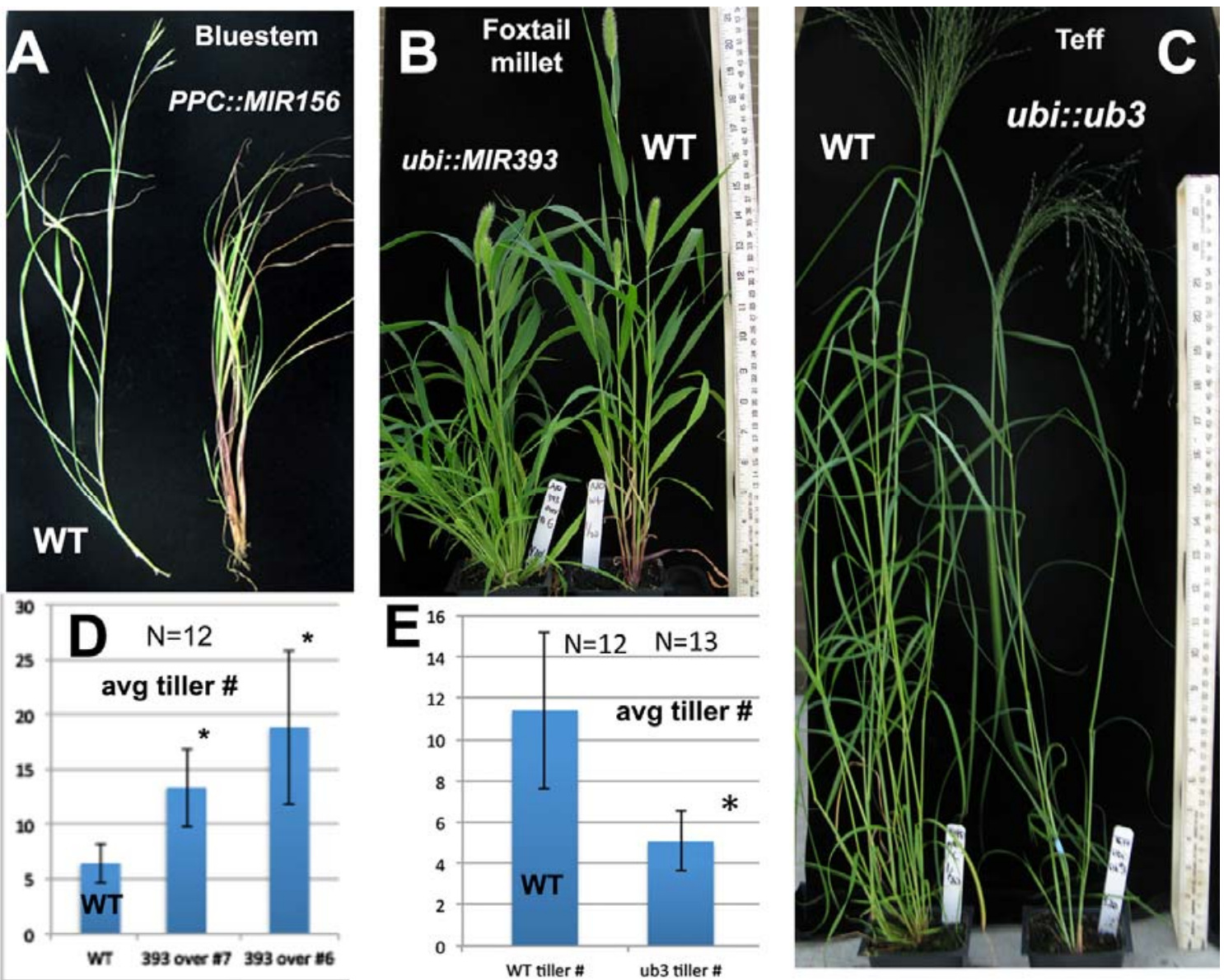

Figure 2 - Analysis of different grass transformants

1A) Comparison of Bluestem MIR156 overexpression transformants to wild type. 1B) Comparison of Setaria viridis MIR393 overexpression transformants to wild type. 1C) Comparison of $u b 3$ teff overexpression transformants to wild type. 1D) Average tiller number in MIR393 overexpressers in Setaria compared to wild type. 1E) Average tiller number in unbranched3 overexpressers in teff compared to wild type. 
BARD Final report Eshed and Hake

We used our transformation protocol to test the hypothesis that temporally regulated microRNAs derived from our previous small RNA sequencing project in switchgrass might be useful to increase axillary branch formation, a trait that could be useful for increasing biomass levels in biofuel crops. We focused on MIR393, which targets auxin hormone receptors and is up-regulated upon the adult phase transition. Overexpression of MiR393 precursor genes demonstrated that tiller branching is significantly increased by overexpression in transgenic Setaria (Fig 2B, D). This construct will be transformed into bluestem and switchgrass to determine if the same effect can be observed in the field.

The converse of the above experiment was done using microRNA target genes instead of microRNAs. We hypothesized that by overexpressing a target of MIR56, we could create the opposite of the extra branching phenotype seen in (Fig 2A) and instead cause reduced branching. This trait could be important for enhancing the agronomic properties of orphan African grain crops.

Depite being grown for centuries and feeding millions of people each year, many orphan African grain crops are in desperate need of genetic improvement. These crops, including teff and millet, are drought and flooding tolerant, display better water usage, and are nutritionally superior to maize. Despite this, they have several drawbacks, including low grain yields and modest forage quality. These problems often result from overproduction of tillers that drain valuable resources away from the main shoot, as well as increased lodging that causes seed loss.

To decrease tillering in teff we overexpressed the $u b 3$ gene that we previously showed to be a tillering repressor that functions redundantly with $u b 2$ and tsh4 (Fig. 1C). Although only two transformants overexpressing $u b 3$ were isolated (Fig. 2C), both showed a $50 \%$ decrease in tillering (Fig. 2E), demonstrating the utility of this approach. We are currently testing these transformants for seed quality and lodging resistance.

\section{C) miR156 links CK signaling with tuberization and flowering}

We recently demonstrated that expression of tomato LOG1 directs suppression of flowering, primarily manifested by the restoration of the indeterminate habit to the otherwise determinate $s p$ shoots as well as the formation of mini tubers from basal axillary buds (Eviatar-Ribak et al., 2013). While 35S:miR156 tomato plants do not form tubers, 35S:LOG1/+ 35S:miR156/+ plants exhibited augmented pigmentation, precocious branching and, most significantly, mini tubers were generated at every nodal bud along all shoots (Fig. 3). Thus, by extending the juvenile stage, miR156 seems to enhance the tuber-forming potential of tomato meristems. Moreover, these findings suggest that, given the right conditions, each axillary meristem in tomato can respond to increased CK signals by a regulated swelling. These results prompted the examination of miR156 in potato. In potato, basal axillary meristems hosted by "juvenile" leaves develop tuber-forming stolons (Fig. 3). Amazingly, under inducing conditions, i.e. short days and low light, aerial axillary buds along the shoots of 35S:miR156 transgenic potato plants generated aerial stolons that terminated with mini-tubers (Fig. 3), while also inducing underground tuberization. Thus, pleiotropic phenotypes of the TLOG1 syndrome and its interaction with the miR156 reveal previously unknown links between cytokinin signaling, flowering and tuberization. 


\section{Figure 3: miR156 links tubers, stolon formation and flowering}

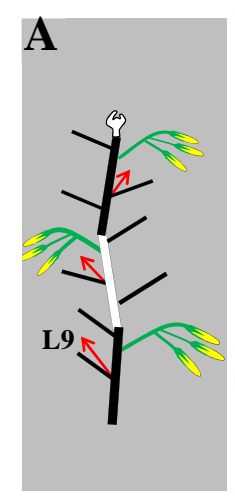

wt

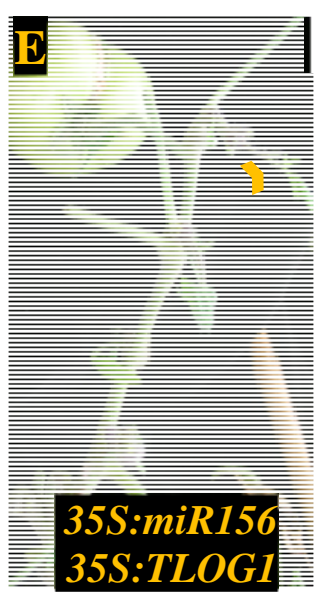

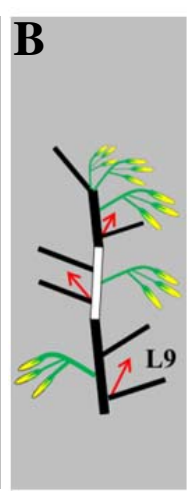

sp
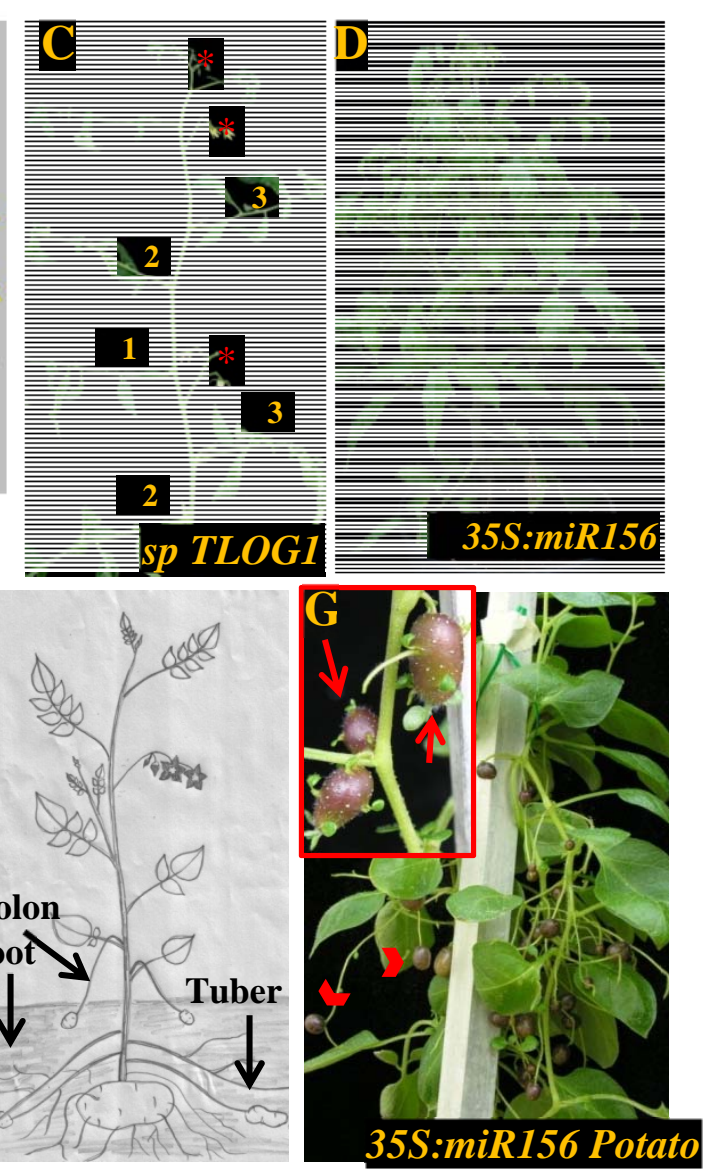

Figure 3 miR156 link tubers, stolon formation and flowerings A, B) Schematic images of WT and self-pruning shoots respectively. C) TLOG1 induces 'indeterminate' flowering shoots and small inflorescences in sp 35S: TLOG1 plants. Note the regular spacing of inflorescences, and the delayed lateral branching at the nodes below inflorescences. D) Excessive branching and delayed flowering in tomato plants with high miR156 levels. E) 35S:miR156 promotes acropetal expansion of mini tuber initiation in TLOG1 shoots. F) A scheme of a potato plant showing the gradual change in leaf complexity, the negative gravitropism of stolons and the axillary buds of basal and distal leaves. G) miR156 induces aerial stolons (arrow in inset) with terminal tubers (arrowhead) in the axills of simple leaves in potato.

Next, we tested the potential of other CK biosynthesis genes to promote tuber formation, and found that fine manipulation of the IPT gene, the rate-limiting factor in CK biosynthesis could also stimulate tuber formation. Strikingly, expression of IPT with the primordia specific driver $p L F S$ resulted in tuber formation on the leaf rachis, at sites where leaflets are connected. This result further highlights the role of CK in stimulating strong sink tissue, but only upon transient upregulaton. 


\section{D) Tomato mutants with delayed sympodial termination can boost field performance.}

One of the striking characteristics of tomato plants expressing 35S:miR156 is the delayed flowering in sympodial buds, those that form after reproductive transition (Figure 3). We therefore looked for mutants with similar characteristics, and generally termed them suppressors of self pruning (Figure 4). The underlying mutants turned out to encode components of the Florigen signaling, a weak allele of $s f t$, the tomato florigen encoding gene, and independent alleles of the SPGB1, a component of the florigen-activating complex. These mutants were used to tune tomato shoot architecture, allowing a mild delay in shoot termination, and as a consequence, higher yields (Park et al., 2014).

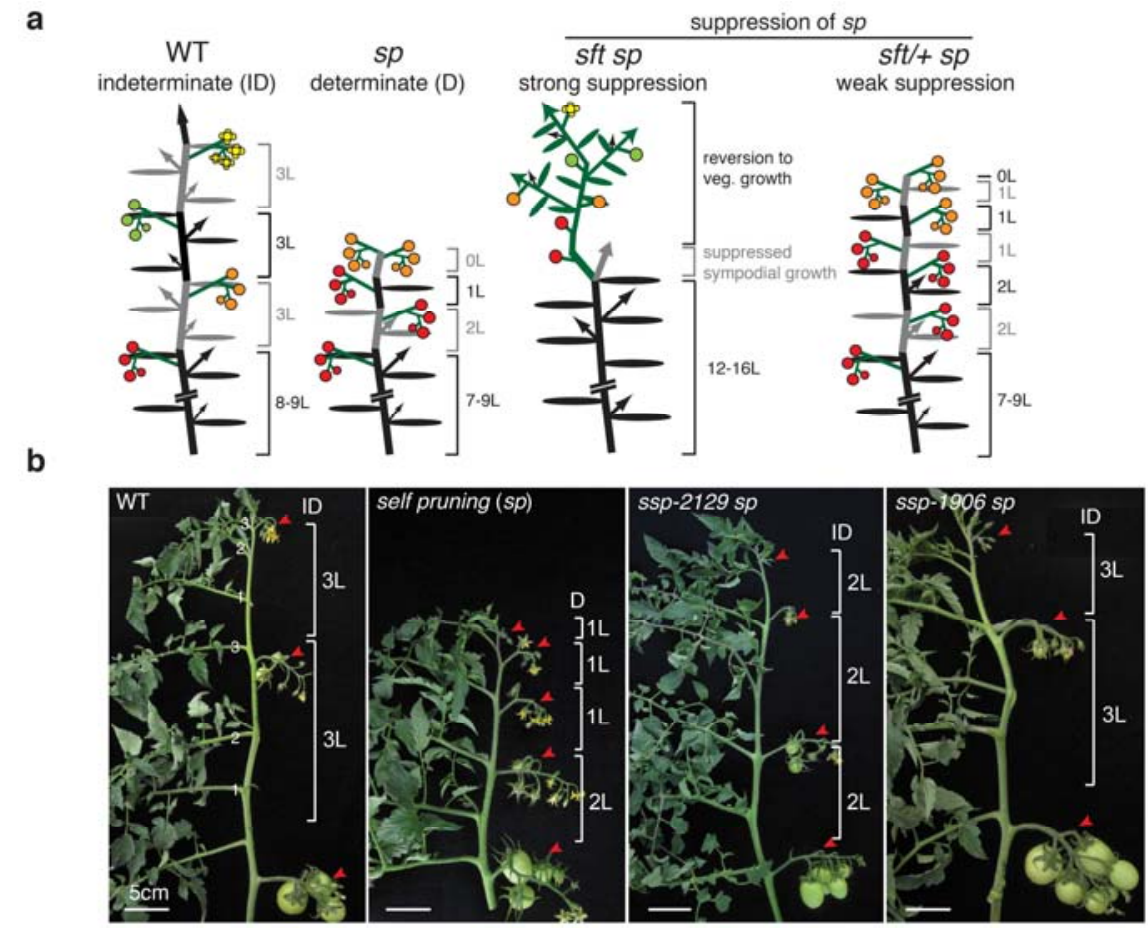

Figure 4. The tomato suppressor of sp (ssp) mutants restore indeterminate growth (a) Diagrams depicting indeterminate wild type (WT) tomato plant architecture, the determinate self pruning ( $s p$ ) mutant, and the strong and weak suppression of $s p$ caused by the single flower truss ( $s f t$ ) mutant in the homozygous ( $s f t s p)$ and heterozygous state $(s f t /+s p)$, respectively. Black bar and flattened ovals from bottom indicate primary shoot and associated leaves. Alternating grey and black bars and ovals reflect successive sympodial shoots. Arrows, canonical axillary shoots; green lines, inflorescences. Note that the weak suppression of $s p$ caused by $s f t /+$ heterozygosity results in a few more sympodial shoots and inflorescences compared to $s p$. (b) Representative main shoots from WT, sp, and the ssp-2129 $s p$ and ssp-1906 sp mutants, which restore indeterminate growth. Three-month-old plants are shown. Arrowheads, inflorescences; L, leaves.

\section{E) Characterization of tomato side shoot transcriptome}

Deep RNA sequencing is democratizing molecular genetics, since detailed expression studies no longer rely on microarray platforms available for only a few standard species and strains. Both of our labs take advantage of the new Illumina instruments and services that include initial data analysis (filtering, normalization, counts per gene etc.). We currently obtain 200- 


\section{BARD Final report Eshed and Hake}

250 million 60-80 bp reads per flow cell lane. In our experience, 20x coverage of the transcriptome provides plenty of power for analysis of even rare transcripts.

To characterize the differences between the primary apical meristem (that forms during embryogenesis), the basal lateral shoot that forms in the axial of a juvenile leaf and a distal lateral shoot that forms in the axil of an adult leaf, we collected apical meristems from all three. The primary shoot meristem (PSM) is gradually formed from the apical part of the developing zygote. Axillary meristems initiate at the boundary of leaf primordia and the stem to form side shoots (SS). Both types of meristems will generate stem, leaves and flowers. Each meristem on the same plant will differentiate into flowers at different times, even though the flowering hormone florigen is systemic and emitted from mature leaves. To uncover the molecular mechanisms underlying the differences between the two meristem types an RNA Expression Dataset of primary and side shoots was constructed from RNA of Primary Shoot Meristem (PSM) and 3 types of Side Shoot meristems (SS); leaf axil -1, -3 and +3 (Figure 5). In broad terms, side shoots have high expression of members that belong to several groups of transcription factors (TF) such as TCP and SPL that may repress SS growth. In addition, several TFs from MADS box family, that include multiple homeotic regulators, show

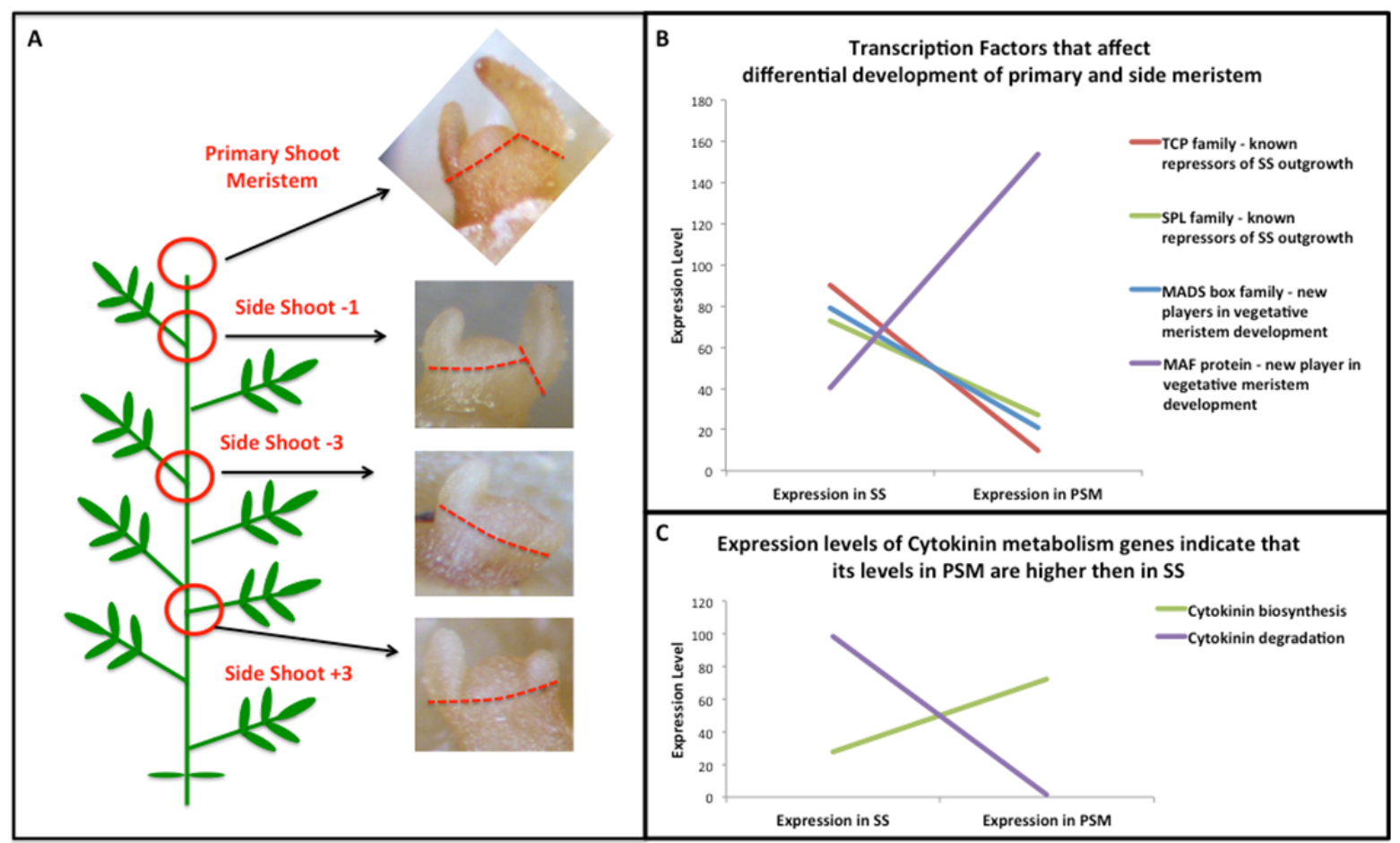

Figure 5. Construction and analysis of RNA Expression Dataset of primary and side shoot meristems.

A. The Expression Dataset was constructed from RNA samples that were extracted from several types of meristems, such as, Primary Shoot Meristem (PSM), and 3 types of Side Shoot meristems (SS), that were originated at leaf axil $-1,-3$ and +3 .

B. In the Dataset we identified several groups of transcription factors. TFs from TCP and SPL families are known SS growth repressors. We found a group of TFs from MADS box family with similar pattern to TCP and SPL except for one MAF protein from MADS family that has reverse expression pattern than its family members.

C. We have identified differences in expression pattern of several genes that belong to Cytokinin metabolism.

quantitative differences between the two meristem classes (B). Likewise, clear differences in 


\section{BARD Final report Eshed and Hake}

expression of genes directing Cytokinin metabolism (C) were evident. Could these represent novel components that differentiate meristem fate? We are currently generating custom-made mutant lines to test these hypotheses.

\section{Details of cooperation:}

The primary goal of our joint project is to examine the possible contribution of age dependent programs to favored shoot architecture and horticultural performance. In addition, we aimed to identify "universal" regulators of side shoots organization that allow them to respond in a different way than the primary shoot to internal and external cues. To that end, the Israeli group characterized side shoots of tomato while the PGEC group analyzed buds of grasses, initially maize, and more recently, Brachypodium. We exchanged the gene lists that came up from the different experiments, and came to the conclusion that several processes are modified in these highly divergent side shoots. Of these, it was clearly evident that TB1/BRC1 factors of the TCP family stand out. Other candidates that were common in both systems were regulation of the GA hormone. However, in each experimental system, different genes were modified. We thus jointly decided to characterize the dynamics of the side shoot in greater details, a program that both labs are still working on. In pararlel, both groups tested the filed performance of selected genotypes where the shoots were modieds either by afe dependent factors or flowering time regulators.

In 2013 the Israeli PI met PhD students from the PGEC and both discussed their recent findings regarding hormonal regulation of apical meristems. Given the extensive amount of data generated by both labs, a joint meeting of the two PI took place in Vermont at the spring of 2015. Moreover, the US PI, Sara Hake will visit Rehovot in the spring of 2016 to present her recent findings. Regardless, throughout the project information was regularly exchanged. We are aware that BARD expects to see joint publications out of our collaboration, however, the research on two different plant families made it difficult to combine results into a coherent study. However, it may be feasible in the coming future to summarize our findings in the form of a review article that draw broader conclusions provided by our wide taxonomic perspectives. 